\title{
The correlation between fractional amplitude of low- frequency fluctuation-based resting-state functional magnetic resonance imaging and facial emotion recognition ability in patients with first-episode schizophrenia
}

\section{Qijie Kuang}

Affiliated Brain Hospital of Guangzhou Medical University: Guangzhou Huiai Hospital

Yi Liu

Affiliated Brain Hospital of Guangzhou Medical University: Guangzhou Huiai Hospital

Sumiao Zhou

Affiliated Brain Hospital of Guangzhou Medical University: Guangzhou Huiai Hospital

Taiyong Bi

Zunyi Medical University

Lin Mi

Affiliated Brain Hospital of Guangzhou Medical University: Guangzhou Huiai Hospital

Shenglin She

Affiliated Brain Hospital of Guangzhou Medical University: Guangzhou Huiai Hospital

Yingjun Zheng ( $\sim$ brainzheng@gzhmu.edu.cn )

Affiliated Brain Hospital of Guangzhou Medical University: Guangzhou Huiai Hospital https://orcid.org/0000-0002-6336-4827

\section{Research Article}

Keywords: First-episode Schizophrenia (FSZ), Visual search, Fractional amplitude of low frequency fluctuation (fALFF), Facial emotion recognition (FER)

Posted Date: October 28th, 2021

DOI: https://doi.org/10.21203/rs.3.rs-919680/v1

License: (c) (i) This work is licensed under a Creative Commons Attribution 4.0 International License.

Read Full License 


\section{Abstract}

Our aim was to analyse the correlation between the fractional amplitude of low-frequency fluctuation (fALFF) and facial emotion recognition (FER) ability in patients with first-episode schizophrenia (FSZ). A total of 28 patients with FSZ and 33 healthy controls (HCs) completed visual search tasks for FER ability. Regions of interest (ROIs) related to facial emotion were obtained from a previous meta-analysis. Pearson correlation analysis was performed to understand the correlation between fALFF and FER ability. Our results indicated that the patients performed worse than the HCs in the accuracy performances of happy FER and fearful FER. The previous meta-analysis results showed that the brain regions related to FER included the bilateral amygdala (AMY)/hippocampus (HIP), right fusiform gyrus (FFG), and right supplementary motor area (SMA). Pearson correlation showed that the fALFF of the right FFG was associated with high-load fearful FER accuracy $(r=-0.43, p=0.022)$. Multiple regression analysis showed that the fALFF of the right FFG was an independent contributor to fearful FER accuracy. Our study indicates that FER ability is correlated with resting-state intrinsic activity in brain regions related to facial emotion, which may provide a reference for the study of FER in schizophrenia.

\section{Highlights}

1. Deficits in facial emotion recognition (FER) ability were evaluated by a visual search task among first-episode schizophrenia (FSZ).

2. FER accuracy, including happy stimulus and fearful stimulus were different between FSZ patients and healthy controls (HC). However, FER reaction time (RT), including happy stimulus and fearful stimulus were not different of them.

3. Multiple regression analysis included gender, age, education, grey matter volume (GMV) and clinical symptoms points as potential confounders.

4. The fALFF of the right FFG was an independent contributor to fearful FER accuracy (low-load task with R2 $=0.255$; high-load task with R2 $=0.524$ ).

\section{Introduction}

Schizophrenia is characterized by various clinical symptoms, such as hallucinations, delusions, speech or behaviour disturbance, and cognitive deficits (Marder and Cannon 2019). Schizophrenia affects approximately $1 \%$ of the general population and carries a significant burden for the individual, family, and wider society (Evensen et al. 2016).

In recent years, more attention has been given to social cognition in Schizophrenia. Social cognition refers to a variety of cognitive processes involved in the accurate perception of the dispositions and intentions of others (Green et al. 2015). Bora et al. conducted a meta-analysis and showed that social cognition deficits were consistent among patients with Schizophrenia (including acute and chronic phases) and persisted even as other clinical symptoms were alleviated, which suggested that they might constitute a marker of Schizophrenia (Bora and Pantelis 2013). Moreover, facial emotion recognition 
(FER) is one of the most studied processes of social cognition in Schizophrenia. Unfortunately, FER was found to be impaired in patients with Schizophrenia (Gao et al. 2021; Kolavarambath et al. 2020). Another study showed that deficits in FER were stable across different stages of the disorder and were already present in first-episode schizophrenia (FSZ) patients (McCleery et al. 2016). In addition, evidence suggests that FER ability is an independent determinant of the prognosis of social cognitive outcomes in Schizophrenia (Green et al. 2012). Recently, She et al. (She et al. 2017) found that the FER cognition of patients with FSZ was impaired in a visual search task, but the visual search function of graphics did not demonstrate any abnormalities, which suggested that facial perception deficits might be a specific marker for Schizophrenia. In addition, a meta-analysis indicated that FER deficits might be useful predictors of Schizophrenia (Martin et al. 2020). Taken together, these studies suggested that FER is one of the core features of Schizophrenia and contributes to poor social functioning.

To date, an extensive body of magnetic resonance imaging (MRI) research has been conducted to explore the changes in processing facial emotion in patients with Schizophrenia. The results of structural MRI studies have shown that the grey matter volume (GMV) of the frontal (particularly the medial and inferior portions), temporal lobes and amygdala (AMY)/ hippocampus (HIP) are related to the facial emotion cognitive function of patients with FSZ (Pera-Guardiola et al. 2016; Rossion et al. 2012). For functional MRI (fMRI), a meta-analysis demonstrated brain activity changes across widespread brain regions when processing facial expressions of emotions, including the AMY, the fusiform gyrus (FFG), the HIP, the superior, medial and dorsolateral frontal cortices and the subcortical structures, which were weaker in patients with Schizophrenia than in HCs (Li et al. 2010; Taylor et al. 2012). Notably, both the GMV and neural activity in the FFG were lower in Schizophrenia patients than in HCs (Kanwisher and Yovel 2006), which is termed the fusiform face area (FFA) (Tarr and Gauthier 2000). FFA was found to be crucial in FER.

Although numerous fMRI studies have examined brain activation during FER, few studies have examined the correlation between intrinsic brain activity (IBA) and FER in patients with Schizophrenia. IBA is associated with dynamic neural and metabolic activity and plays a key role in brain function (Raichle 2015). The amplitude of low-frequency fluctuation (ALFF), which is one method used to analyse intrinsic brain activity, can directly demonstrate blood oxygenation level-dependent (BOLD) signals and reflect the idiopathic activity levels of neurons in voxels according to their energy levels in the resting state (Zang et al. 2007). The fractional ALFF (fALFF) is the normalized ALFF, which can be used to improve the sensitivity and specificity in detecting spontaneous brain activity by effectively minimizing the influence of physiological noise (Zou et al. 2008). However, numerous studies have examined FER deficits in patients with Schizophrenia, but the association between FER deficits and Schizophrenia is still not well understood. Recently, Luo et al. revealed the genetic-brain-cognition mediation pathway in patients with Schizophrenia by the GMV and fALFF methods, suggesting that the fALFF can be used to represent the IBA and can be used for studying cognition in Schizophrenia (Luo et al. 2018).

To analyse the correlation between resting-state intrinsic activity in brain regions related to facial emotion and FER ability in patients with FSZ, we adopted the fALFF method and visual search task to measure 
brain region intrinsic activity and FER ability. We hypothesized that the fALFF in brain regions related to facial emotion would be related to FER ability in patients with Schizophrenia. Only FSZ patients were included to avoid the confounding factors of duration of illness and antipsychotics drug use.

\section{Materials And Methods Participants}

FSZ patients were selected based on the following inclusion criteria: 1) Must have normal or corrected-tonormal vision; 2) Completely satisfied the DSM-IV (Diagnostic and Statistical Manual of Mental Disorders, Fourth Edition) criteria for diagnosis of Schizophrenia, wherein the structured clinical interview for DSM-IV (SCI-D) was conducted by two trained psychiatrists with more than 5 years of experience; 3 ) Psychiatric symptoms are assessed on the Positive and Negative Syndrome Scale (PANSS) (Kay et al. 1987), which requires a score of over 50 points; 4) Must be experiencing their first episode, have a duration of psychosis of no more than 2 years and have been on antipsychotic medication for no more than 6 months before participating in this study; and 5) Had no history of electroconvulsive therapy (ECT) within six months. The exclusion criteria were as follows: 1) other psychotic or affective disorders, mental retardation, drug dependence, organic brain lesions, and/or physical diseases; and 2) contraindications for magnetic resonance imaging (MRI). Thirty-one patients with FSZ met the inclusion criteria to participate in the study, but three patients were excluded after failing to complete the psychophysical experiment. HCs were selected based on the following inclusion criteria: 1) gender-, age- and educationmatched to the patient group; and 2) no history of mental illness and no family history of mental illness. Exclusion criteria were the same as the exclusion criteria for the patient group. Finally, $33 \mathrm{HC}$ participants finished our study. This study was approved by the Institutional Review Board of the Affiliated Brain Hospital of Guangzhou Medical University, and all subjects provided informed written consent for participation.

\section{Behaviour experiment}

The experimental procedure was similar to the procedure described in a previous study (She et al. 2017). Each participant completed two visual search experiments to assess facial ER ability, and the process is shown in Fig. 1. In the experiments, all subjects were asked to complete the happy FER experiment first and then the fearful FER experiment. The experimental procedure is as follows. Firstly, a small white cross present at the centre of the monitor lasted $1000-2000 \mathrm{~ms}$. Subsequently, two or four faces are presented for $600 \mathrm{~ms}$, included or excluded emotional faces. Emotional faces are presented in half of the trials. It is worth mentioning that all the figures used during the experiment were selected from the Chinese Facial Affective Picture System (CFAPS) (Gong et al. 2011), including thirty-two happy faces, thirty-two fearful faces and sixty-four neutral faces. The air, ears, and face contour of all faces were excluded via Photoshop. Emotional faces are presented in half of the trials. Once faces disappears, subjects need to judge as quickly and accurately as possible (" $\mathrm{n}$ " if it contains an emotional face, or " $\mathrm{m}$ " if it does not). Next, the experimental procedure begins to cycle and the small white cross present again in 
the centre of the monitor. Each FER experiment contains six blocks and each block contains 40 trials. Adequate rest periods between two experiments were ensured in order to obtain the best results. Worthy of mention, all subjects were given appropriate instruction and some practice to understand the experiment beforehand.

\section{Imaging acquisition and processing}

Upon completion of the psychophysical experiment, magnetic resonance data collection was performed on the same day. The collection of imaging data was performed in the magnetic resonance chamber of the radiology department by an attending level or higher professional technician from the radiology department. All subjects were scanned with a Philips 3.0T MRI system, and the signals were received by a standard head coil. During the scan, subjects were asked to secure their heads with supporting foam pads and to minimize the noise with rubber ear buds.

Whole-brain rs-fMRI scans comprising a total of 240 echo-planar imaging (EPI) volumes were obtained with the following parameters: repetition time $(T R)=2000 \mathrm{~ms}$, echo time $(T E)=30 \mathrm{~ms}$, flip angle $=90^{\circ}$, matrix $=64 \times 64$, slice thickness $=4 \mathrm{~mm}$, slice number $=33$, voxel size $=3.44 \times 3.44 \times 4.60 \mathrm{~mm}$, slice gap, and field of view $(F O V)=192 \mathrm{~mm} \times 192 \mathrm{~mm}$. Rs-fMRI data were analysed and processed with RESTPLUS (Jia et al. 2019) in MATLAB 2013b. The preprocessing included removal of the first ten dummy scans, slice timing correction, realignment, spatial standardization (normalized to Montreal Neurological Institute (MNI) space by using the conventional EPI template), smoothing, detrending, and nuisance covariate regression.

To calculate fALFF, the fast Fourier transform (FFT) of the time series of the whole-brain signal was calculated first, converting the signal into a frequency domain power spectrum. Then, the square root of the power spectrum at each frequency was averaged across the filtered band $(0.01-0.08 \mathrm{~Hz})$ to obtain the ALFF of the signal. The fALFF was obtained by dividing the ALFF by the full-band amplitude. The fALFF of each voxel was then divided by the global mean of the fALFF (mfALFF) to eliminate differences in whole-brain fALFF at the overall level between individuals. Finally, the mfALFF signal of the ROIs was extracted by the RESTPLUS Toolbox (Jia et al. 2019).

High-resolution 3D-T1 structural images were collected following the rs-fMRI scan. The T1 structure images were obtained using a planar imaging sequence of gradient echoes with the following parameters: $\mathrm{TR}=8.2 \mathrm{~ms}, \mathrm{TE}=3.8 \mathrm{~ms}$, flip angle $=7$ degrees, matrix $=256 \times 256$, thickness $=1.0 \mathrm{~mm}$, scanning layers $=165$. Voxel-based morphometry (VBM) was employed to explore the GMVs of different brain regions in the two groups. The preprocessing of the 3D- $\mathrm{T}_{1} \mathrm{MRI}$ was performed with the SPM8 subtool (http://www.fil.ion.ucl.ac.uk/spm/software/spm8) and included the following steps: segmentation, spatial standardization, and smoothing. Then, the GMVs of the ROls were extracted by the RESTPLUS Toolbox (Jia et al. 2019).

\section{ROI acquisition}


A series of meta-analyses of human fMRI data were downloaded using Neurosynth (www.neurosynth.org; (Yarkoni et al. 2011)) to identify brain regions associated with FER ability. Neurosynth, a platform that automatically synthesizing and analyses the results of many different neuroimaging studies, and a list of search terms are generated after integration and classification of high-frequency words. Neurosynth was used to select the ROIs for our study with search items related to facial emotion. "Emotional faces" and "facial expressions" were selected as the keywords for searching the fMRI series. The areas of overlap within both domains using the imaging calculator from SPM 12 (http://www.fil.ion.ucl.ac.uk/spm/software/spm12) were used as ROls ( $p<0.01$, FDR corrected).

\section{Statistical analysis}

All values of subjects were indicated by mean (SD), except for gender. Double sample $t$-tests and chisquare tests were used to compare the basic demographic characteristics between the two groups. Average accuracy and correct reaction time (RT) were considered the behavioural results. The psychophysical experiment was a 2 (stimulus: happy/fearful faces) $\times 2$ (condition load: 2 or 4 items) $\times 2$ (subject group: Schizophrenia or HC) mixed design, in which condition load and stimulation were withinsubject factors and subject group (Schizophrenia or $\mathrm{HC}$ ) was a between-subject factor. If there was an interaction between stimulus, group or condition load, a simple effects analysis was performed to compare the differences in accuracy or correct RT between two groups, or if not, a multiple $t$-test with Bonferroni adjustment was performed instead. The effect size (ES) was provided for each statistical test. Pearson correlation analysis was used to analyse the correlation between FER ability and the fALFF of the ROIs. Multiple regression models were used to quantify the amount of variance in FER ability explained by the fALFF of ROIs related to facial emotion after controlling for several potential confounders. The confounders included gender, age, education, GMV of ROIs and clinical symptoms shown on PANSS. All statistical tests were conducted as two-tailed tests with 0.05 as the level of significance (a). The statistical analyses were performed using SPSS 22.0.

\section{Results}

\section{Demographic and clinical characteristics}

Finally, a total of twenty-eight patients and thirty-three HC participants finished our study. There were no significant differences in items of gender $\left(\chi^{2}=0.46, p=0.500\right)$, age $(t=0.59, p=0.557)$ or education ( $t=$ $0.42, p=0.674$ ) between the two groups. The basic demographic and descriptive characteristics of the participants are shown in Table 1. 
Table 1

Clinical, demographic characteristics and FER ability of the participants

\begin{tabular}{|c|c|c|c|}
\hline & $\begin{array}{l}\text { Patients with FSZ } \\
(n=28)\end{array}$ & $\begin{array}{l}\text { HCs } \\
(n=33)\end{array}$ & $x^{2} / t$ \\
\hline Gender (male/female) ${ }^{a}$ & $16 / 12$ & $16 / 17$ & $0.46^{b}$ \\
\hline Age (years) & $25.14(6.87)$ & $24.24(5.03)$ & 0.59 \\
\hline Education (years) & $11.32(3.26)$ & $11.67(3.11)$ & 0.42 \\
\hline Duration of illness (months) & $8.71(7.19)$ & $\mathrm{N} / \mathrm{A}$ & $\mathrm{N} / \mathrm{A}$ \\
\hline Antipsychotics drug use $(\mathrm{mg})^{a}$ & $217.38(230.41)$ & $\mathrm{N} / \mathrm{A}$ & $\mathrm{N} / \mathrm{A}$ \\
\hline PANSS positive symptoms & $17.36(5.18)$ & $\mathrm{N} / \mathrm{A}$ & $\mathrm{N} / \mathrm{A}$ \\
\hline PANSS negative symptoms & $13.61(4.25)$ & $\mathrm{N} / \mathrm{A}$ & $\mathrm{N} / \mathrm{A}$ \\
\hline PANSS psychopathology symptoms & $34.14(8.77)$ & $\mathrm{N} / \mathrm{A}$ & $\mathrm{N} / \mathrm{A}$ \\
\hline PANSS total & $65.11(15.48)$ & $\mathrm{N} / \mathrm{A}$ & $\mathrm{N} / \mathrm{A}$ \\
\hline Low-load happy stimulus accuracy & $0.92(0.06)$ & $0.96(0.05)$ & $2.75^{\star}$ \\
\hline High-load happy stimulus accuracy & $0.85(0.07)$ & $0.91(0.07)$ & $3.62^{\star \star}$ \\
\hline Low-load fearful stimulus accuracy & $0.77(0.12)$ & $0.89(0.07)$ & $4.70^{\star \star}$ \\
\hline High-load fearful stimulus accuracy & $0.71(0.09)$ & $0.83(0.08)$ & $5.30^{\star \star}$ \\
\hline Low-load happy stimulus correct RT & $0.42(0.24)$ & $0.36(0.25)$ & 0.83 \\
\hline High-load happy stimulus correct RT & $0.57(0.34)$ & $0.45(0.23)$ & 1.59 \\
\hline Low-load fearful stimulus correct RT & $0.52(0.24)$ & $0.42(0.20)$ & 1.77 \\
\hline High-load fearful stimulus correct RT & $0.60(0.27)$ & $0.51(0.24)$ & 1.44 \\
\hline \multicolumn{4}{|c|}{$\begin{array}{l}\text { Values are presented as mean (s.d.). N/A, not applicable; PANSS, Positive and Negative Syndrome } \\
\text { Scale; FSZ, First-episode schizophrenia; HCs, Healthy controls; RT, Reaction time. }\end{array}$} \\
\hline \multicolumn{4}{|c|}{${ }^{a}$ equivalent to Chlorpromazine dosage; ${ }^{\text {b }}$ Chi-square test. } \\
\hline${ }^{*} . p<0.05 ; * \star . p<0.01$ & & & \\
\hline
\end{tabular}

\subsection{Behavioural performance of the visual search task}

In the psychophysical experiment, trials with a correct RT less than $3 \mathrm{~s}$ were included for further analysis. The average accuracy and correct RT were then calculated for each condition (Table 1). 
Regarding the accuracy results, only the interaction between stimulus and subject group was significant, $F=10.96, p=0.001, E S=0.044$, indicating that the FER accuracy in Schizophrenia patients was affected differently by stimuli than in HCs. Simple effect analysis showed that the group effect remained significant after controlling for the stimulus (happy face: $F=13.57, p<0.001, \mathrm{ES}=0.054$; fearful face: $F=$ $69.97, p<0.001, E S=0.226$ ), while the stimulus effect was significant after controlling for the group (patient group: $F=90.89, p<0.001$, ES $=0.276$; HC group: $F=29.86, p<0.001, \mathrm{ES}=0.111$ ). Significant main effects were observed for condition load, $F=36.64, p<0.001, \mathrm{ES}=0.133$, which indicated a lower accuracy for the 4-item load. Multiple $t$-tests showed that the accuracy for each condition was significant (happy/2 items: $t=2.75, p=0.032$, ES $=0.706$; happy $/ 4$ items: $t=3.62, p=0.004$, ES $=0.931$; fearful $/ 2$ items: $t=4.70, p<0.001$, ES $=1.208$, fearful $/ 4$ items: $t=5.30, p<0.001$, ES $=1.362$ ), indicating that the FER ability of patients with FSZ was impaired compared to the FER ability of the control group.

Regarding the correct RT results, none of the interactions among the abovementioned factors was significant, indicating that the FER correct RT of the two groups were not affected differently by stimulus or load. However, the main effects were significant for load, $F=9.69, p=0.002$, ES $=0.039$, which indicated that the correct RT was longer for the 4-item load experiment but was similar for the different stimuli for both loads of subjects. The correct RT of each condition was not significant (happy/2 items: $t$ $=0.83, p=1.000, \mathrm{ES}=0.214$; happy $/ 4$ items: $t=1.59, p=0.464$, ES $=0.410$; fearful $/ 2$ items: $t=1.77, p=$ 0.328 , ES $=0.455$; fearful $/ 4$ items: $t=1.44, p=0.628$, ES $=0.369$ ), which indicated that the correct RT of the patients was similar to the RT of the HCs for each condition.

\section{ROls related to facial emotion}

The collection of meta-analysis data using Neurosynth terminated on February 1, 2020. One hundred and 250 studies from the automated meta-analysis survived for the search terms "emotional faces" and "facial expressions", respectively. The brain regions for the search term "emotional faces" included mainly the bilateral AMY, bilateral HIP, and bilateral FFG. The brain regions for the search term "facial expressions" included mainly the bilateral AMY, bilateral HIP, right FFG, left inferior temporal gyrus, right inferior occipital gyrus, right triangular part of the inferior frontal gyrus, right superior temporal gyrus, and right middle temporal gyrus. The ROls, formed from the area of overlap across the two domains related to facial emotions, included the bilateral AMY, bilateral HIP, right FFG, and right SMA. The results are described in detail in Table 2, and an illustration is given in Fig. 2 . 
Table 2

ROIs related to facial emotions.

\begin{tabular}{|c|c|c|c|c|c|}
\hline \multirow[t]{2}{*}{ Brain region(AAL) } & \multicolumn{3}{|c|}{ Peak MNI coordinates } & \multirow[t]{2}{*}{ BA } & \multirow{2}{*}{$\begin{array}{l}\text { Cluster size } \\
\text { (voxels) }\end{array}$} \\
\hline & $\mathbf{x}$ & $\mathbf{y}$ & $\mathbf{z}$ & & \\
\hline Left AMY/ Left HIP & -30 & -6 & -28 & - & 484 \\
\hline Right AMY/ Right HIP & 34 & -6 & -28 & - & 416 \\
\hline Right FFG & 42 & -42 & 24 & 37 & 45 \\
\hline Right SMA & 6 & 14 & 68 & - & 11 \\
\hline
\end{tabular}

All brain regions are thresholded at $p<0.01$, FDR corrected, with a minimum cluster size of 10 voxels. $\mathrm{MNI}$ coordinates of ROls are reported here.

AAL, Anatomical Automatic Labeling; MNI, Montreal Neurological Institute; BA, Brodmann area; AMY, amygdala;HIP, hippocampus; FFG, fusiform gyrus; SMA, supplementary motor area.

\section{Correlation results}

Pearson correlation showed that the fALFF of the right FFG was associated with high-load fearful FER accuracy $(r=-0.43, p=0.022)$ and had a correlation trend with low-load fearful FER accuracy $(r=-0.34, p$ $=0.077)$. The results are described in Table S1. Further multiple regression analysis showed that the fALFF of the FFG was an independent contributor to fearful FER accuracy (low-load task with $\mathrm{R}^{2}=0.255$ (beta $=-0.40, t=-2.29, p=0.034)$ ); high-load task with $\mathrm{R}^{2}=0.524$ (beta $\left.=-0.46, t=-3.30, p=0.004\right)$ ). In further, we found the GMV of the FFG was also an independent contributor to high-load fearful FER accuracy (beta $=-0.46, t=-3.08, p=0.006$ ). The results are described in detail in Table 3 . However, no significant correlation was found between the correct RT and the fALFF of ROIs, and the results are described in Table S1. 
Table 3

Multiple regression analysis result of patient group.

\begin{tabular}{|c|c|c|c|c|c|c|c|c|}
\hline \multirow[b]{2}{*}{ Variable } & \multicolumn{4}{|c|}{ Low-load fearful FER accuracy } & \multicolumn{4}{|c|}{ High-load fearful FER accuracy } \\
\hline & B & $\beta$ & $t$ & $95 \% \mathrm{Cl}$ & B & $\beta$ & $t$ & $95 \% \mathrm{Cl}$ \\
\hline Gender & 0.02 & 0.10 & 0.53 & $(-0.07,0.12)$ & -0.02 & -0.13 & -0.82 & $\begin{array}{l}(-0.09 \\
0.04)\end{array}$ \\
\hline Age & 0.00 & -0.27 & -1.40 & $(-0.01,0.00)$ & 0.00 & -0.29 & -1.90 & $\begin{array}{l}(-0.01 \\
0.00)\end{array}$ \\
\hline Education & 0.00 & -0.08 & -0.45 & $(-0.02,0.01)$ & 0.00 & -0.05 & -0.35 & $\begin{array}{l}(-0.01 \\
0.01)\end{array}$ \\
\hline PANSS & 0.00 & 0.09 & 0.45 & $(0.00,0.00)$ & 0.00 & 0.24 & 1.59 & $(0.00,0.00)$ \\
\hline GMV of Right FFG & -0.49 & -0.28 & -1.49 & $(-1.18,0.20)$ & -0.67 & -0.46 & $-3.08^{\star \star}$ & $\begin{array}{l}(-1.12 \\
-0.21)\end{array}$ \\
\hline $\begin{array}{l}\text { fALFF of Right } \\
\text { FFG }\end{array}$ & -0.47 & -0.40 & $-2.29^{\star}$ & $\begin{array}{l}(-0.90 \\
-0.04)\end{array}$ & -0.45 & -0.46 & $-3.30^{* *}$ & $\begin{array}{l}(-0.73, \\
-0.16)\end{array}$ \\
\hline
\end{tabular}

\section{Discussion}

Facial emotions represent a complex stimulus that conveys a variety of messages that are important for social interaction. Our study revealed that patients with FSZ had worse FER accuracy for fear than FER accuracy for happiness. To our knowledge, this is the first MRI study to explore the relationship between the fALFF of brain regions related to facial emotion and FER ability in patients with Schizophrenia. Furthermore, as previous studies have apparently determined the effect of brain region GMV on FER function, our study controlled for GMV factors. We observed the relationship between FER ability and the fALFF of the right FFG in FSZ patients. Our findings suggest that FER ability is correlated with restingstate intrinsic activity in some brain regions related to facial emotion.

In the behavioural test, we adopted a visual search task to behaviourally measure the FER ability in Schizophrenia, which is more concerned with the detection and identification of stimuli compared with the match-to-sample task (Lin et al. 2013). Regarding accuracy, similar to the findings of most previous studies, our test showed that patients with FSZ performed worse in FER than HCs (Bosnjak Kuharic et al. 2019; Zhang et al. 2018). Furthermore, only the interaction between stimulus and group was significant, which indicated that the accuracy of FER in Schizophrenia was impaired more severely for fearful facial emotion than happy facial emotion. Previous studies showing that in Schizophrenia, negative FER (Mitrovic et al. 2020; Romero-Ferreiro et al. 2016), including fearful emotion, was more severe than positive FER. Similar results were found in FER research of patients with FSZ. Bonfils et al. (Bonfils et al. 2019) showed that patients with FSZ had the highest accuracy in recognizing happy facial emotion and the least accuracy in recognizing fearful facial emotion, which suggested FSZ patients had more 
difficulty in identifying fearful facial emotion. Regarding the correct RT, our results showed that the difference between the two groups was not significant, although the RTs of the patients with Schizophrenia were longer than those of the HCs for each condition. This result was different from the result of a previous study, which indicated that disgusted FER in patients with Schizophrenia had a longer RT than the RT in HCs (Yang et al. 2018). The reasons for the inconsistent results might contribute to the possibility of differences in stimuli and differences in the tasks used. The main effect consequences for both accuracy and correct RT suggested better performance on the low-load task than on the high-load task, which was similar to the results of another previous study by our group (She et al. 2017).

The bilateral AMY, bilateral HIP, right FFG, and right supplementary motor area (SMA) were preserved after analysis. Previous MRI findings have shown detailed intricate connection of the FFG with the HIP and AMY, which appear to underpin the network that processes facial emotion (Smith et al. 2009). As the core brain region of facial emotion cognition, the function of the AMY is similar in Schizophrenia, as indicated in a meta-analysis showing reduced AMY activation in response to facial emotion processing in Schizophrenia (Gur et al. 2002). Maher et al. concluded that patients with Schizophrenia had significantly lower neural activity by activation in the AMY fearful and happy faces (Maher et al. 2016). Similar results were obtained by Wang et al. in Schizophrenia patients with high negative symptoms (Wang et al. 2018), and they found decreased functional connectivity between the AMY and the ventral medial prefrontal cortex and dorsolateral prefrontal cortex. Additionally, patients with AMY damage showed reduced emotional response in the FFA during the recognition of facial expressions (Vuilleumier et al. 2004).

A negative correlation was found between altered right FFG MRI measurements and fearful FER accuracy in patients with FSZ, especially altered mfALFF of the right FFG. A large number of studies have shown that the FFG is associated with FER in different disorders, including bipolar disorder (Bertocci et al. 2019), autism spectrum disorder (Kovarski et al. 2019), and traumatic brain injury (Rigon et al. 2019). The FFG, especially the right FFG, supports face processing, social communication, and facial identity processing (Pourtois et al. 2010). In previous studies of structural MRI, Zhang et al. found that GMV of the FFG was related to FER in Schizophrenia (Zhang et al. 2018). A similar result was found by Jung et al. (Jung et al. 2021), who found that GMV in the FFG was positively correlated with FER and emotional intensity recognition, but to date, no study on the relationship between fALFF and FER in Schizophrenia has been conducted. Although our test found this correlation, the underlying neural mechanism of the FFG in FER is still not understood. One group hypothesized that the FFG plays a crucial role in face recognition but not facial expression (Axelrod and Yovel 2015), which is the fundamental element of face processing. FMRI research showed functional disconnection between the visual cortex and right FFA in Schizophrenia (Maher et al. 2019). Another study hypothesized that the fusiform face area was also involved in processing emotional expressions (Harry et al. 2013). Multivoxel pattern-analysis approaches to functional neuroimaging data have shown that emotion categories could be decoded from responses to facial expressions in the FFG (Brooks et al. 2019). Unfortunately, neither AMY nor HIP was found to be correlated with FER accuracy in the visual search task. Two reasons might cause this result. First, evidence suggests that AMY includes two functional subregions, the basolateral AMY and centromedial AMY (Mahan and Ressler 2012). Our test extracted only the fALFF signal of AMY, which might be a 
confounding factor for the result. The other reason might be the possibility of the differences in the behaviour experiment used.

However, there has been little previous research on FER RT. Some studies have shown that hippocampal brain volume in older adults is positively correlated with the speed of correct FER (Szymkowicz et al. 2016). Another study including patients with FSZ showed that RT for a high degree of disgust FER task was longer than $\mathrm{HCs}$ and increased activation of the left and right middle temporal gyrus and right caudate in response to the high degree of disgust stimulus (Yang et al. 2018). However, they did not conduct a correlation analysis to examine the relationship between prolonged RT and activated brain regions. Therefore, the relationship between RT of FER and the intrinsic activity of the resting-state in brain regions needs further investigation.

\section{Limitations}

The comparatively small sample size is a limitation of our study. Furthermore, stimuli should be examined in further studies because our test included only fearful faces as negative stimuli. In addition, fALFF was used to assess intrinsic brain activity in this study. Other methods, such as ReHo and functional connectivity, should be used in further studies to better understand the underlying neural mechanism between Schizophrenia and FER ability.

\section{Conclusions}

In summary, our study indicates a degree of impairment in the accuracy of happy and fearful FER in the FSZ. Furthermore, our study indicates that FER ability is correlated with resting-state intrinsic activity in the brain regions that are related to facial emotion. This correlation is altered in patients with FSZ, which may provide a reference for the study of FER in Schizophrenia.

\section{Declarations}

Acknowledgements This work was supported by the Planed Science and Technology Projects of Guangzhou (201904010267).

Appendix A. Supporting information Supplementary data associated with this article can be found in the online version.

Conflict of interest None of the authors have a conflict of interest to declare.

\section{References}

Axelrod, V., \& Yovel, G. (2015). Successful decoding of famous faces in the fusiform face area. PLoS One, 10(2), e0117126. 
Benson, P. J., Beedie, S. A., Shephard, E., Giegling, I., Rujescu, D., \& St Clair, D. (2012). Simple viewing tests can detect eye movement abnormalities that distinguish schizophrenia cases from controls with exceptional accuracy. Biological psychiatry, 72(9), 716-724.

Bertocci, M. A., Hanford, L., Manelis, A., lyengar, S., Youngstrom, E. A., Gill, M. K., et al. (2019). Clinical, cortical thickness and neural activity predictors of future affective lability in youth at risk for bipolar disorder: initial discovery and independent sample replication. Molecular psychiatry, 24(12), 1856-1867.

Bonfils, K. A., Ventura, J., Subotnik, K. L., \& Nuechterlein, K. H. (2019). Affective prosody and facial emotion recognition in first-episode schizophrenia: Associations with functioning \& symptoms. Schizophrenia research Cognition, 18, 100153.

Bora, E., \& Pantelis, C. (2013). Theory of mind impairments in first-episode psychosis, individuals at ultrahigh risk for psychosis and in first-degree relatives of schizophrenia: systematic review and metaanalysis. Schizophrenia research, 144(1-3), 31-36.

Bosnjak Kuharic, D., Makaric, P., Kekin, I., Lukacevic Lovrencic, I., Savic, A., Ostojic, D., et al. (2019). Differences in Facial Emotional Recognition Between Patients With the First-Episode Psychosis, Multiepisode Schizophrenia, and Healthy Controls. Journal of the International Neuropsychological Society: JINS, 25(2), 165-173.

Brooks, J. A., Chikazoe, J., Sadato, N., \& Freeman, J. B. (2019). The neural representation of facialemotion categories reflects conceptual structure. Proceedings of the National Academy of Sciences of the United States of America, 116(32), 15861-15870.

Evensen, S., Wisloff, T., Lystad, J. U., Bull, H., Ueland, T., \& Falkum, E. (2016). Prevalence, Employment Rate, and Cost of Schizophrenia in a High-Income Welfare Society: A Population-Based Study Using Comprehensive Health and Welfare Registers. Schizophrenia Bulletin, 42(2), 476-483.

Gao, Z., Zhao, W., Liu, S., Liu, Z., Yang, C., \& Xu, Y. (2021). Facial Emotion Recognition in Schizophrenia. Frontiers in psychiatry, 12, 633717.

Gong, X., Huang, Y.X., Wang, Y., \& Luo, Y.J. (2011). Revision of the Chinese facial affective picture system. Chinese Mental Health Journal, 25, 40-46.

Green, M. F., Bearden, C. E., Cannon, T. D., Fiske, A. P., Hellemann, G. S., Horan, W. P., et al. (2012). Social cognition in schizophrenia, Part 1: performance across phase of illness. Schizophrenia Bulletin, 38(4), 854-864.

Green, M. F., Horan, W. P., \& Lee, J. (2015). Social cognition in schizophrenia. Nature reviews Neuroscience, 16(10), 620-631.

Gur, R. E., McGrath, C., Chan, R. M., Schroeder, L., Turner, T., Turetsky, B. I., et al. (2002). An fMRI study of facial emotion processing in patients with schizophrenia. The American journal of psychiatry, 159(12), 
Harry, B., Williams, M. A., Davis, C., \& Kim, J. (2013). Emotional expressions evoke a differential response in the fusiform face area. Frontiers in human neuroscience, 7, 692.

Jia, X.Z., Wang, J., Sun, H.Y., Zhang, H., Liao, W., Wang, Z., et al. (2019). RESTplus: an improved toolkit for resting-state functional magnetic resonance imaging data processing. Science Bulletin, 64, 953-954.

Jung, S., Kim, J. H., Kang, N. O., Sung, G., Ko, Y. G., Bang, M., et al. (2021). Fusiform gyrus volume reduction associated with impaired facial expressed emotion recognition and emotional intensity recognition in patients with schizophrenia spectrum psychosis. Psychiatry research Neuroimaging, 307, 111226.

Kanwisher, N., \& Yovel, G. (2006). The fusiform face area: a cortical region specialized for the perception of faces. Philosophical transactions of the Royal Society of London Series B, Biological sciences, 361(1476), 2109-2128.

Kay, S. R., Fiszbein, A., \& Opler, L. A. (1987). The positive and negative syndrome scale (PANSS) for schizophrenia. Schizophrenia bulletin, 13(2), 261-276.

Kolavarambath, R., Sudhir, P. M., Prathyusha, P. V., \& Thirthalli, J. (2020). Emotion Recognition, Emotion Awareness, Metacognition, and Social Functioning in Persons with Schizophrenia. Indian journal of psychological medicine, 42(2), 147-154.

Kovarski, K., Mennella, R., Wong, S. M., Dunkley, B. T., Taylor, M. J., \& Batty, M. (2019). Enhanced Early Visual Responses During Implicit Emotional Faces Processing in Autism Spectrum Disorder. Journal of autism and developmental disorders, 49(3), 871-886.

Li, H., Chan, R. C., McAlonan, G. M., \& Gong, Q. Y. (2010). Facial emotion processing in schizophrenia: a meta-analysis of functional neuroimaging data. Schizophrenia bulletin, 36(5), 1029-1039.

Lin, I. M., Fan, S. Y., Huang, T. L., Wu, W. T., \& Li, S. M. (2013). The Associations between Visual Attention and Facial Expression Identification in Patients with Schizophrenia. Psychiatry investigation, 10(4), 393398.

Luo, N., Sui, J., Chen, J., Zhang, F., Tian, L., Lin, D., et al. (2018). A Schizophrenia-Related Genetic-BrainCognition Pathway Revealed in a Large Chinese Population. EBioMedicine, 37, 471-482.

Mahan, A. L., \& Ressler, K. J. (2012). Fear conditioning, synaptic plasticity and the amygdala: implications for posttraumatic stress disorder. Trends in neurosciences, 35(1), 24-35.

Maher, S., Ekstrom, T., \& Chen, Y. (2016). Impaired visual cortical processing of affective facial information in schizophrenia. Clinical psychological science : a journal of the Association for Psychological Science, 4(4), 651-660. 
Maher, S., Ekstrom, T., Ongur, D., Levy, D. L., Norton, D. J., Nickerson, L. D., et al. (2019). Functional disconnection between the visual cortex and right fusiform face area in schizophrenia. Schizophrenia research, 209, 72-79.

Marder, S. R., \& Cannon, T. D. (2019). Schizophrenia. The New England journal of medicine, 381(18), 1753-1761.

Martin, D., Croft, J., Pitt, A., Strelchuk, D., Sullivan, S., \& Zammit, S. (2020). Systematic review and metaanalysis of the relationship between genetic risk for schizophrenia and facial emotion recognition. Schizophrenia research, 218, 7-13.

McCleery, A., Lee, J., Fiske, A. P., Ghermezi, L., Hayata, J. N., Hellemann, G. S., et al. (2016). Longitudinal stability of social cognition in schizophrenia: A 5-year follow-up of social perception and emotion processing. Schizophrenia research, 176(2-3), 467-472.

Mitrovic, M., Ristic, M., Dimitrijevic, B., \& Hadzi Pesic, M. (2020). Facial Emotion Recognition and Persecutory Ideation in Paranoid Schizophrenia. Psychological reports, 123(4), 1099-1116.

Pera-Guardiola, V., Contreras-Rodriguez, O., Batalla, I., Kosson, D., Menchon, J. M., Pifarre, J., et al. (2016). Brain Structural Correlates of Emotion Recognition in Psychopaths. PLoS One, 11(5), e0149807.

Pourtois, G., Spinelli, L., Seeck, M., \& Vuilleumier, P. (2010). Modulation of face processing by emotional expression and gaze direction during intracranial recordings in right fusiform cortex. Journal of cognitive neuroscience, 22(9), 2086-2107.

Raichle, M. E. (2015). The restless brain: how intrinsic activity organizes brain function. Philosophical transactions of the Royal Society of London Series B, Biological sciences, 370(1668).

Rigon, A., Voss, M. W., Turkstra, L. S., Mutlu, B., \& Duff, M. C. (2019). Functional neural correlates of facial affect recognition impairment following TBI. Brain imaging and behavior, 13(2), 526-540.

Romero-Ferreiro, M. V., Aguado, L., Rodriguez-Torresano, J., Palomo, T., Rodriguez-Jimenez, R., \& PedreiraMassa, J. L. (2016). Facial affect recognition in early and late-stage schizophrenia patients. Schizophrenia research, 172(1-3), 177-183.

Rossion, B., Hanseeuw, B., \& Dricot, L. (2012). Defining face perception areas in the human brain: a largescale factorial fMRI face localizer analysis. Brain and cognition, 79(2), 138-157.

She, S., Zhang, B., Li, X., Zhang, X., Li, R., Li, J., et al. (2017). Face-related visual search deficits in firstepisode schizophrenia. Psychiatry research, 256, 144-149.

Smith, C. D., Lori, N. F., Akbudak, E., Sorar, E., Gultepe, E., Shimony, J. S., et al. (2009). MRI diffusion tensor tracking of a new amygdalo-fusiform and hippocampo-fusiform pathway system in humans. Journal of magnetic resonance imaging : JMRI, 29(6), 1248-1261. 
Szymkowicz, S. M., Persson, J., Lin, T., Fischer, H., \& Ebner, N. C. (2016). Hippocampal Brain Volume Is Associated with Faster Facial Emotion Identification in Older Adults: Preliminary Results. Frontiers in aging neuroscience, 8, 203.

Tarr, M. J., \& Gauthier, I. (2000). FFA: a flexible fusiform area for subordinate-level visual processing automatized by expertise. Nature neuroscience, 3(8), 764-769.

Taylor, S. F., Kang, J., Brege, I. S., Tso, I. F., Hosanagar, A., \& Johnson, T. D. (2012). Meta-analysis of functional neuroimaging studies of emotion perception and experience in schizophrenia. Biological psychiatry, 71(2), 136-145.

Vuilleumier, P., Richardson, M. P., Armony, J. L., Driver, J., \& Dolan, R. J. (2004). Distant influences of amygdala lesion on visual cortical activation during emotional face processing. Nature neuroscience, $7(11), 1271-1278$.

Wang, Y., Li, Z., Liu, W. H., Wei, X. H., Jiang, X. Q., Lui, S. S. Y., et al. (2018). Negative Schizotypy and Altered Functional Connectivity During Facial Emotion Processing. Schizophrenia bulletin, 44(suppl_2), S491-S500.

Yang, C., Qi, A., Yu, H., Guan, X., Wang, J., Liu, N., et al. (2018). Different levels of facial expression recognition in patients with first-episode schizophrenia: A functional MRI study. General psychiatry, 31(2), e000014.

Yarkoni, T., Poldrack, R. A., Nichols, T. E., Van Essen, D. C., \& Wager, T. D. (2011). Large-scale automated synthesis of human functional neuroimaging data. Nature Methods, 8(8), 665-670.

Zang, Y. F., He, Y., Zhu, C. Z., Cao, Q. J., Sui, M. Q., Liang, M., et al. (2007). Altered baseline brain activity in children with ADHD revealed by resting-state functional MRI. Brain \& development, 29(2), 83-91.

Zhang, X., Yao, J., Lv, Y., Zhao, X., Li, Y., Sui, Y., et al. (2018). An Association Study on the Cognitive Function and the Cerebral Grey Matter Volume of Patients with First-Episode Schizophrenia. Shanghai archives of psychiatry, 30(3), 154-167.

Zou, Q. H., Zhu, C. Z., Yang, Y., Zuo, X. N., Long, X. Y., Cao, Q. J., et al. (2008). An improved approach to detection of amplitude of low-frequency fluctuation (ALFF) for resting-state fMRI: fractional ALFF. Journal of neuroscience methods, 172(1), 137-141.

\section{Supplemental Table}

Sup. Table 1 Correlation between mfALFF and FER ability of patient group. 


\begin{tabular}{|lllll|}
\hline & $\begin{array}{l}\text { Left } \\
\text { AMY/ } \\
\text { Left HIP }\end{array}$ & $\begin{array}{l}\text { Right AMY/ Right } \\
\text { HIP }\end{array}$ & $\begin{array}{l}\text { Right } \\
\text { FFG }\end{array}$ & Right SMA \\
\hline Low-load happy stimulus accuracy & 0.21 & 0.07 & 0.1 & 0.24 \\
\hline High-load happy stimulus accuracy & 0.17 & -0.04 & 0.2 & 0.1 \\
\hline Low-load fearful stimulus accuracy & 0.13 & 0.22 & -0.34 & -0.05 \\
\hline High-load fearful stimulus accuracy & 0.26 & 0.25 & $-0.43^{*}$ & 0.13 \\
\hline $\begin{array}{l}\text { Low-load happy stimulus correct RT } \\
\text { High-load happy stimulus correct }\end{array}$ & 0.11 & -0.03 & -0.08 & 0.02 \\
\hline $\begin{array}{l}\text { RT } \\
\text { Low-load fearful stimulus correct }\end{array}$ & -0.03 & -0.02 & -0.11 & -0.04 \\
\hline $\begin{array}{l}\text { RT } \\
\text { RT }\end{array}$ & -0.2 & -0.18 & 0.03 & -0.2 \\
\hline
\end{tabular}

*. $p<0.05$.

\section{Figures}

\section{Stimuli}

(600ms)

\section{target}

a happy face
Fixation (1000-2000ms)

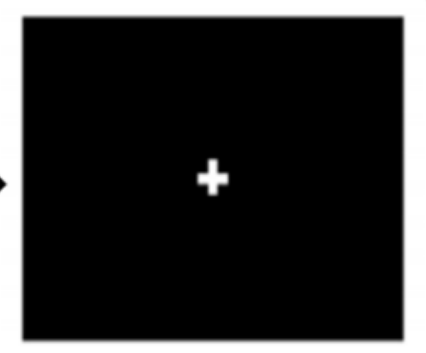

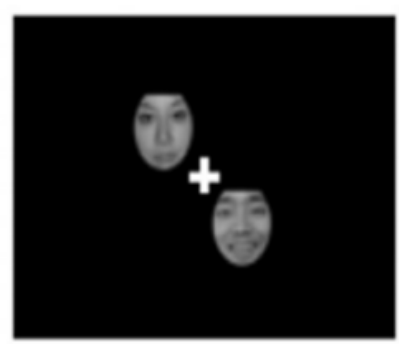

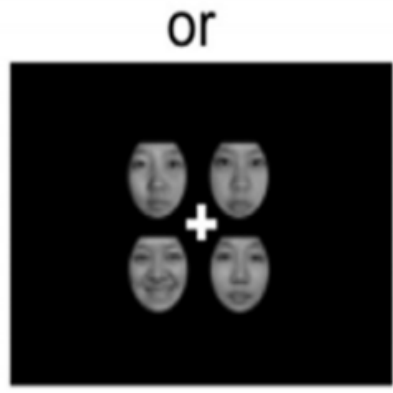

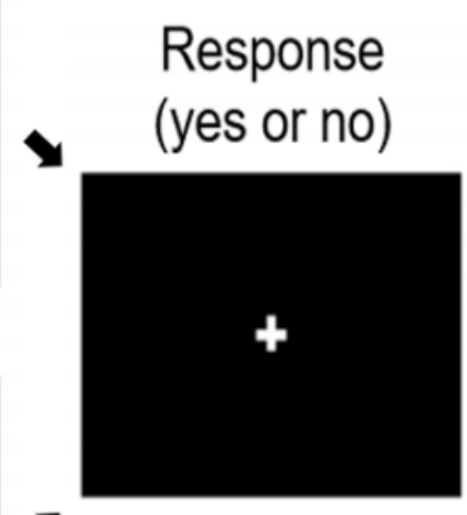


Figure 1

Schematic demonstration of the experimental procedure for happy face search

$\mathbf{L}$
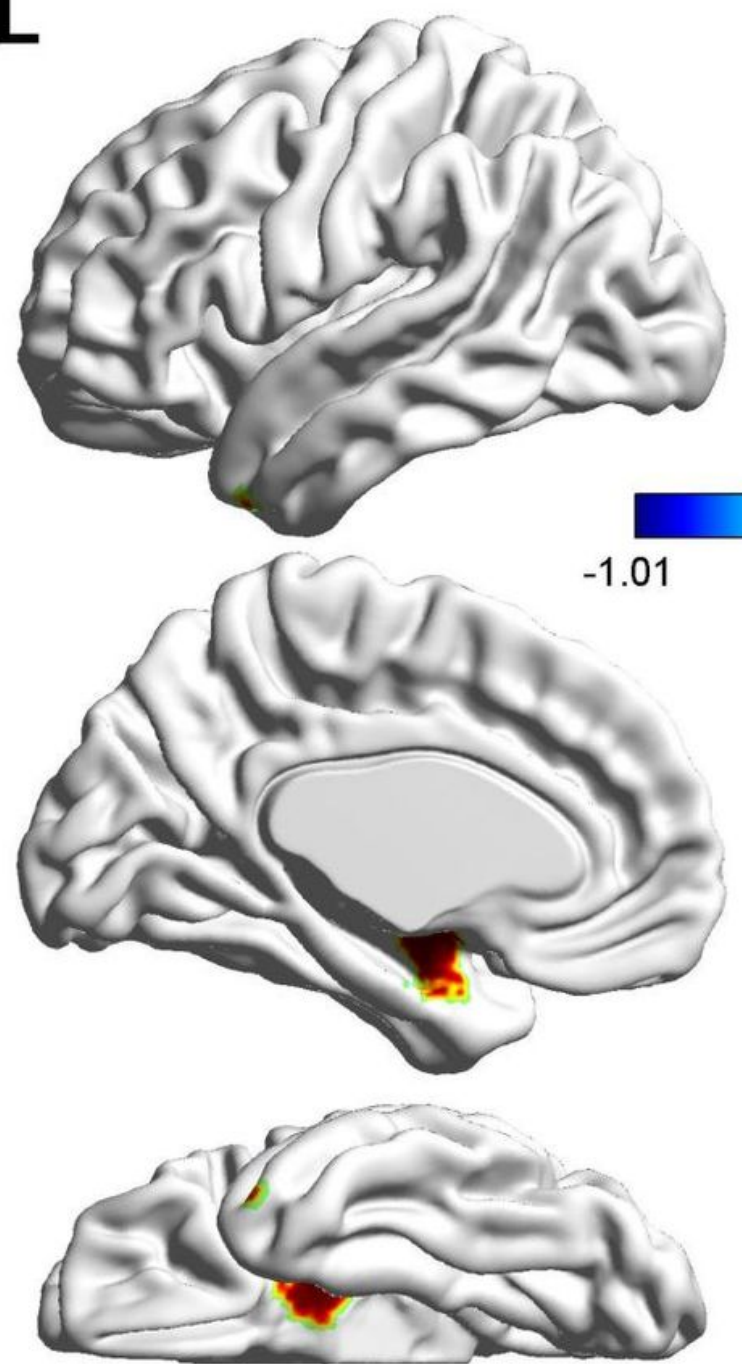

$\mathbf{R}$
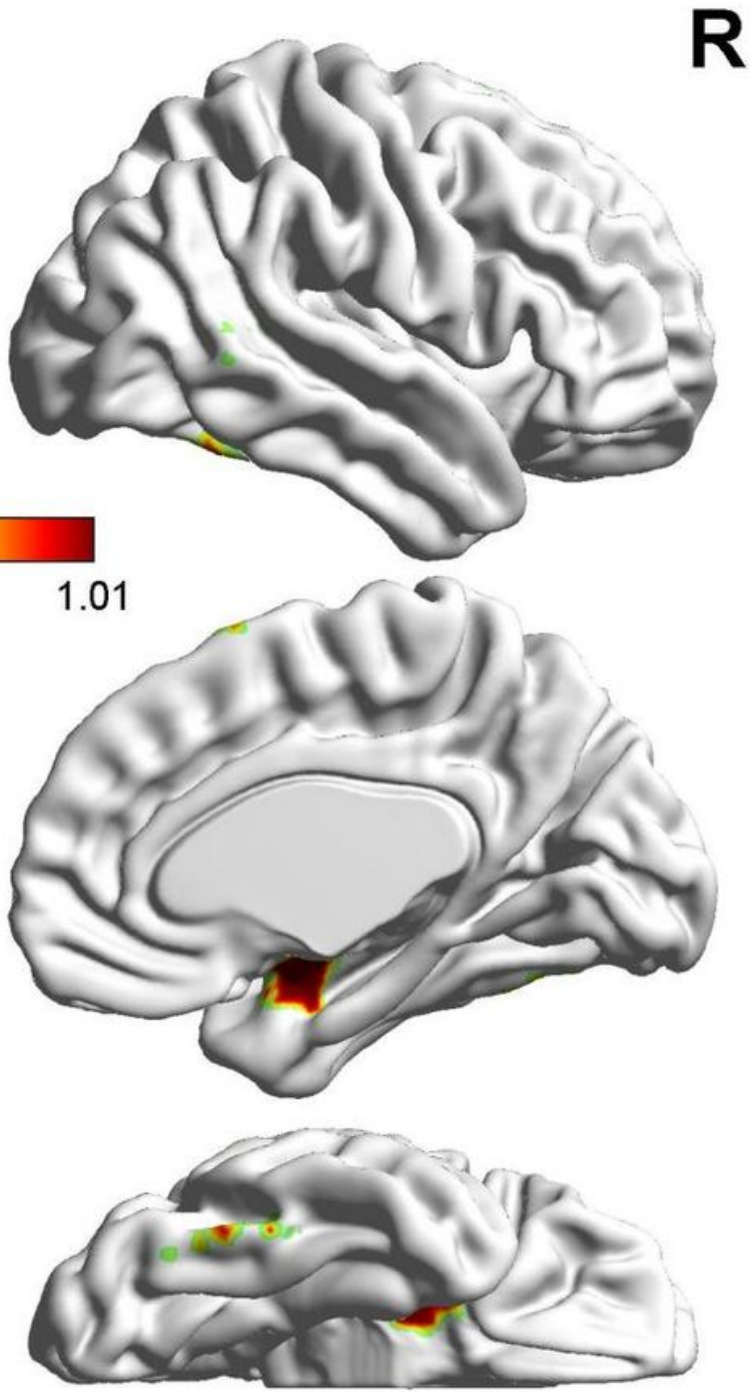

Figure 2

The lateral and medial view of brain regions related to "emotional faces" and "facial expressions" using Neurosynth ( $p<0.01$, FDR corrected, with a minimum cluster size of 10 voxels)

\section{Supplementary Files}

This is a list of supplementary files associated with this preprint. Click to download.

- BIBChecklist.pdf 